\title{
Are Recently Trained Tumor Fellows Performing Less Tumor Surgery? An Analysis of 10 Years of the ABOS Part II Database
}

\author{
Kyle R. Duchman MD, Benjamin J. Miller MD, MS
}

Received: 23 April 2016/ Accepted: 3 August 2016/Published online: 10 August 2016

(C) The Association of Bone and Joint Surgeons (B) 2016

\begin{abstract}
Background The majority of orthopaedic trainees pursue additional subspecialty training at the conclusion of residency. Although national trends indicate that fellowshiptrained surgeons are more frequently performing cases in their defined subspecialties, this may not be the case for recently trained tumor fellows. Prior work has established that low tumor case volume is a significant stressor for recently trained tumor fellows. Given the relative rarity of musculoskeletal tumors, it is important for prospective trainees to have clear expectations for the proportion of specialty-specific procedures early during their careers. In addition, knowledge of anticipated specialty case volume is important to optimize fellowship training and to provide
\end{abstract}

Each author certifies that he, or a member of his immediate family, has no funding or commercial associations (eg, consultancies, stock ownership, equity interest, patent/licensing arrangements, etc) that might pose a conflict of interest in connection with the submitted article.

All ICMJE Conflict of Interest Forms for authors and Clinical Orthopaedics and Related Research ${ }^{\mathbb{R}}$ editors and board members are on file with the publication and can be viewed on request.

Each author certifies that his institution approved or waived approval for the human protocol for this investigation and that all investigations were conducted in conformity with ethical principles of research.

Electronic supplementary material The online version of this article (doi:10.1007/s11999-016-5023-9) contains supplementary material, which is available to authorized users.

K. R. Duchman ( $₫)$, B. J. Miller

Department of Orthopaedics and Rehabilitation, University of

Iowa, 200 Hawkins Drive, 01015 JPP, Iowa City, IA 52242,

USA

e-mail: kyle-duchman@uiowa.edu guidance for meeting the public health requirements for orthopaedic oncology.

Questions/purposes We wished to determine (1) the number of examinees who self-reported tumor fellowship training during the last decade; (2) how many tumor fellowship-educated surgeons did an additional fellowship(s) in other subspecialties; (3) the number and proportion of tumor, trauma, adult reconstruction, and other procedures performed by tumor-trained fellows; and (4) changes in the proportion of procedures performed by tumor-trained fellows during the 10-year period of the study.

Methods The American Board of Orthopaedic Surgery Part II database was used to identify examinees who reported tumor fellowship training between 2004 and 2013. All submitted procedures were broadly categorized as "tumor," "trauma," "adult reconstruction," or "other." Annual procedure volumes were calculated and univariate analysis allowed comparison of categorized procedures during the duration of the study.

Results The median annual number of candidates reporting tumor fellowship training was 12.5 (range, 7-16). There were 28 of 118 (24\%) candidates who reported additional fellowship training. A total of 14,718 procedures were performed by all candidates with tumor fellowship training during the 10-year period of the study, $42 \%$ of which were categorized as tumor procedures. Overall, only $36 \%$ of candidates reported tumor procedures making up greater than $50 \%$ of their case volume. Between 2004 to 2005 and 2012 to 2013, the proportion of tumor procedures decreased $(45 \%$ versus $36 \%$; $p<0.001)$, whereas the number of adult reconstruction procedures increased $(9 \%$ versus $19 \%$; $\mathrm{p}<0.001)$.

Conclusions Between 2004 and 2013, only one-third of recently trained tumor fellows had practices with tumor procedures accounting for greater than $50 \%$ of their total 
Table 1. Additional subspecialty training for candidates who reported multiple fellowships

\begin{tabular}{lr}
\hline Additional subspecialty training* & Number $(\%)$ \\
\hline Adult reconstruction & $10(31)$ \\
Sports & $2(6)$ \\
Hand & $2(6)$ \\
Trauma & $5(16)$ \\
Spine & $7(22)$ \\
Pediatrics & $6(19)$ \\
Years & $5 / 22(23)$ \\
$2004-2005$ & $3 / 20(15)$ \\
$2006-2007$ & $6 / 18(33)$ \\
$2008-2009$ & $4 / 30(13)$ \\
$2010-2011$ & $10 / 28(36)$ \\
$2012-2013$ & \\
\hline
\end{tabular}

* The number of additional fellowships obtained; four candidates obtained more than two fellowships.

case volume. Furthermore, the proportion of tumor cases performed by recently trained tumor fellows decreased during the same time. The proportion of specialty-specific procedures is lower in orthopaedic oncology than other orthopaedic subspecialties, which is important information for current trainees interested in orthopaedic oncology fellowship training and for orthopaedic oncology educators. The findings in this study should serve as an initial platform for further discussion regarding the optimal number of fellowship-trained orthopaedic oncologists required to meet regional and national needs for an accessible and proficient work force.

\section{Introduction}

Nearly $90 \%$ of today's orthopaedic residents will pursue additional subspecialty fellowship training, a number that has continued to increase with time [8, 9]. Potential explanations for the recent increase in fellowship training include the desire for a focused clinical practice, angst resulting from limited exposure to techniques during residency training, market influences, and financial incentives $[7,9]$. Postresidency training is a decision that will reverberate throughout the career of an orthopaedic surgeon, and it is important for orthopaedic trainees to understand how fellowship training may shape their early career practice patterns.

For the last decade, orthopaedic oncology (orthopaedic tumor surgery) has had the lowest annual number of available fellowship positions [5, 6, 9, 18]. Although active members in the Musculoskeletal Tumor Society (MSTS) report that more than $70 \%$ of their time is dedicated to tumor procedures [18], the percentage of time dedicated to tumor procedures for early-career orthopaedic oncologists is notably less [15]. Additionally, a previous survey of early-career orthopaedic oncologists showed that insufficient tumor case volume was consistently reported as a "source of stress" that was not shared by orthopaedic oncologists later in their careers [15]. National trends indicate that, as a whole, fellowship-trained orthopaedic surgeons are more frequently performing cases in their defined subspecialty [9], but this has not been investigated specifically in orthopaedic oncology. Specialty-specific case volume also has important implications, apart from trainee expectations, as greater surgeon and hospital volume are associated with improved quality of care for specialty procedures such as THA and TKA [4, 10, 11]. The goal of fellowship training, from a national perspective, should be to supply the populace with enough specialty providers to ensure timely and reliable access, but not at the cost of diminished surgical prowess or attenuated outcomes. Thus, this topic has broad relevance to those involved in orthopaedic education and resource allocation [17].

The aim of our study was to use the American Board of Orthopaedic Surgeons (ABOS) Part II database to identify fellowship-trained orthopaedic oncologists to determine (1) the number of examinees who self-reported tumor fellowship training during the last decade; (2) how many tumor fellowship-educated surgeons did an additional fellowship(s) in other subspecialties; (3) the number and proportion of tumor, trauma, adult reconstruction, and other procedures performed by tumor-trained fellows; and (4) changes in the proportion of procedures performed by tumor-trained fellows during the 10-year period of the study. 


\section{Materials and Methods}

The ABOS Part II database was used to identify examinees who self-reported orthopaedic oncology fellowship training for cases performed between 2004 and 2013. Data collection methods for the ABOS Part II database have been described $[1,9]$. Briefly, examinees are eligible to sit for Part II after passing the written ABOS Part I examination and after a minimum of 22 months of practice at a single institution. At that point, examinees submit a list of all surgical procedures performed during a 6-month period with a minimum of 35 cases required. For each submitted procedure, various data are collected by the ABOS. For the purpose of this study, we recorded procedure year, Common Procedural Terminology (CPT) codes, ICD-9, and fellowship training history. All data are deidentified, and no long-term outcomes are collected. Candidates sit for the ABOS Part II examination during the year after case submission. Candidates are not longitudinally followed, and as such, each year represents a new group of candidates, with the exception of those who may be retaking the examination. Because ABOS data are deidentified, this study was deemed exempt by the institutional review board at the University of Iowa.

After identifying all examinees with orthopaedic oncology fellowship training, CPT codes were analyzed and checked for accuracy. In the ABOS Part II database, it is not required to assign a primary CPT code for a particular procedure, and multiple codes frequently are submitted. All unique CPT codes listed in the cohort were identified and broadly categorized as "tumor," "trauma," "adult reconstruction," or "other" procedures (Appendix 1. Supplemental material is available with the online version of $\operatorname{CORR}^{\circledR}$.). For the purpose of this study, any

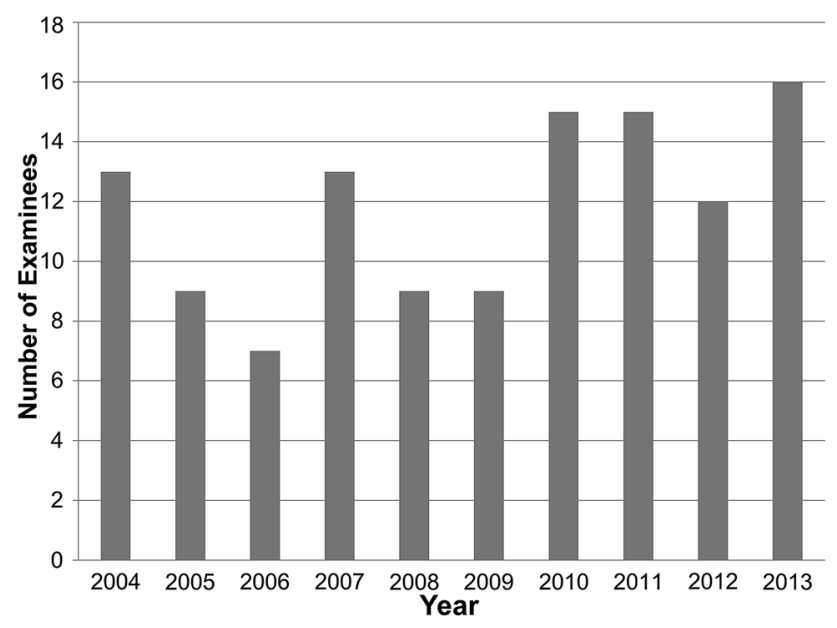

Fig. 1 This graph shows the number of examinees who reported a history of orthopaedic oncology fellowship training between 2004 and 2013. procedure that included at least one CPT code consistent with a tumor procedure was coded as "tumor". CPT codes that did not uniquely define a tumor procedure but are frequently performed in tumor surgery, such as amputation or arthroplasty associated with tumor resection, were further defined by ICD-9 diagnosis codes and classified as "tumor" if the associated diagnosis was a tumor or tumorlike condition.

After categorization, any examinee who did not perform a single tumor procedure in their submitted cases was excluded from analysis because this was thought to represent an error, misinterpretation during data submission, or a candidate who was purposefully not including orthopaedic oncology as part of their clinical practice. A single examinee was excluded for this reason, leaving a total of 118 examinees available for analysis.

\section{Statistical Analysis}

Descriptive statistical methods were used to describe annual and overall procedure volume and proportion of procedures performed in the defined categories. To account for year-to-year variability, procedures performed by examinees in adjacent years were combined (2004-2005, 2006-2007, 2008-2009, 2010-2011, 2012-2013) during several portions of the analysis. Univariate statistical analysis included chi-square and Fisher's exact tests as appropriate, allowing comparison of categorized procedures between years. A $\mathrm{p}$ value less than 0.05 was considered statistically significant. Statistical analysis was performed using SPSS Version 23.0 (IBM Corp, Armonk, NY, USA).

\section{Results}

Among the 118 ABOS Part II examinees with orthopaedic oncology fellowship training, the median annual number of candidates was 12.5 (range, 7-16). The greatest number of examinees performing cases was in 2013 (Fig. 1). During the first half of the study, the median annual number of candidates with orthopaedic oncology fellowship training was nine (range, 7-13), compared with 15 (range, 9-16) for the second half of the study.

Twenty-eight of 118 (24\%) candidates reported multiple fellowships, with adult reconstruction as the most common additional area of training (10/28, 31\%) (Table 1). Although the highest percentage of candidates with multiple fellowships occurred during the final year of the study (8/16 candidates), there was no difference in the percentage of candidates who reported multiple fellowship training 


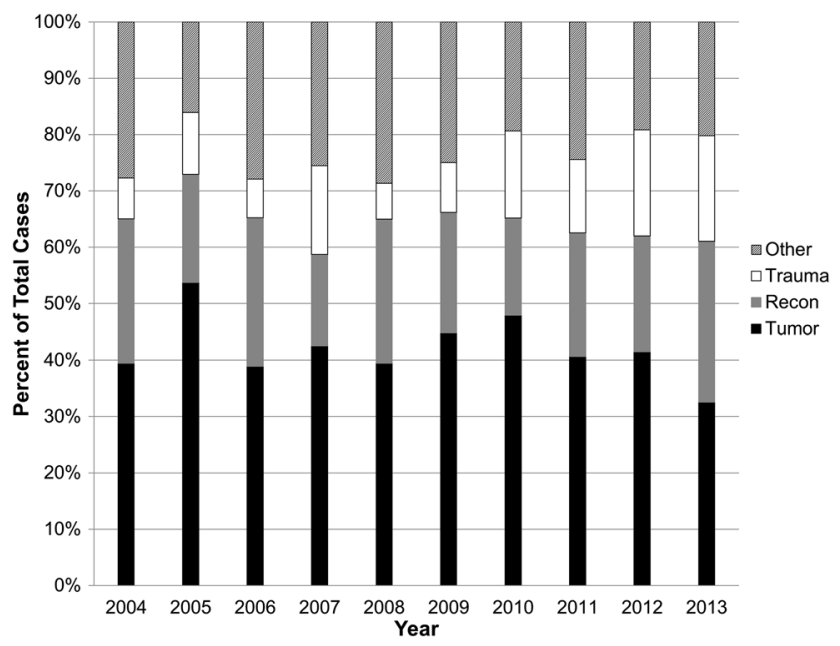

Fig. 2 This graph shows the proportion of tumor, adult reconstruction (recon), trauma, and other procedures performed by examinees who reported a history of orthopaedic oncology fellowship training between 2004 and 2013.

during the first or second half of the study (12/51 [24\%] versus $16 / 67[24 \%] ; \mathrm{p}=0.965$ ).

A total of 14,718 procedures were performed by examinees with orthopaedic oncology fellowship training. Of these, 6113 of $14,718(42 \%)$ cases were categorized as tumor procedures, whereas 3297 of $14,718(22 \%), 1873$ of $14,718(13 \%)$, and 3435 of $14,718(23 \%)$ were categorized as trauma, adult reconstruction, or other procedures, respectively (Fig. 2). In only 3 years, 2005, 2009, and 2012, did tumor procedures make up at least $50 \%$ of the total procedure volume for more than half of the examinees. In these years, six of nine (67\%), five of nine (56\%), and six of $12(50 \%)$ examinees, respectively, had $50 \%$ or more of their total procedure volume attributable to tumor procedures. Overall, only 43 of 118 (36\%) examinees had tumor procedures account for more than $50 \%$ of their total case volume. As a group, candidates who reported multiple fellowships performed a lower proportion of tumor procedures than those with only tumor fellowship training (1253/3408 [37\%] versus 4860/11,310 [43\%]; $p<0.001)$. However, there was no difference in the mean number of tumor procedures reported by multiple or tumor-only fellowship candidates (45 versus 53 cases; $\mathrm{p}=0.152$ ). Additionally, the proportion of candidates with tumor procedures comprising more than $50 \%$ of their total procedure volume did not differ between the two groups (10/ 28 [36\%] versus 32/90 [36\%]; $p=0.998)$.

To account for year-to-year variability, trends were assessed after combining adjacent years. In 2004-2005, the median number of procedures performed by each examinee was 119 (range, 48-203) compared with 128 (range, 38287) in 2012-2013. The median number of tumor

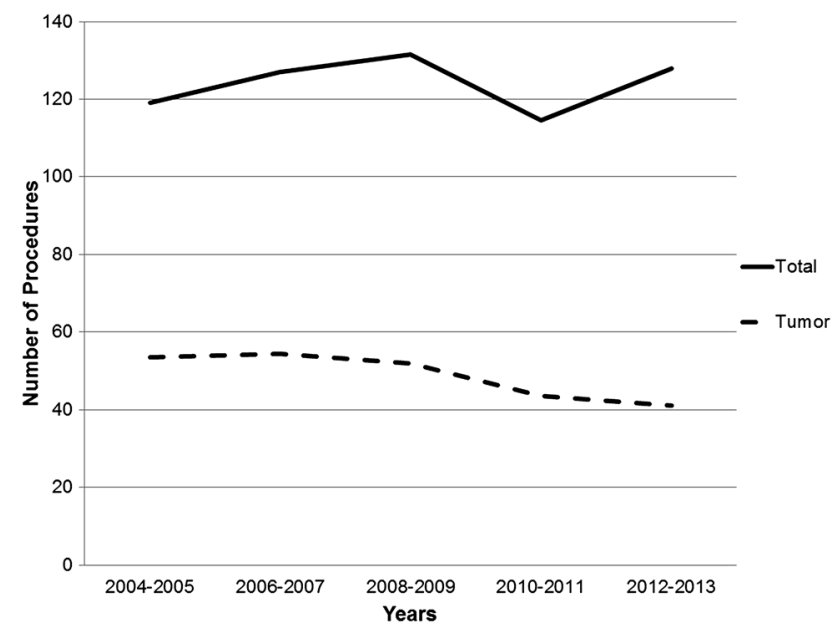

Fig. 3 This graph shows the median number of total procedures and tumor procedures performed by examinees who reported a history of orthopaedic oncology fellowship training. Adjacent years have been combined to account for year-to-year variability and better display trends during the 10 -year period of the study.

procedures performed by each examinee during 2004-2005 was 54 (range, 8-134) compared with 41 (range, 3-84) during 2012 to 2013 (Fig. 3). When comparing the number of tumor procedures performed with all other procedures performed during 2004-2005 and 2012-2013, the proportion of tumor cases performed decreased between the two times (1190/2618 [45\%] versus 1255/3447 [36\%]; p < 0.001) (Fig. 3). This trend was consistent even when excluding candidates with multiple fellowship training from the analysis (946/1971 [48\%] versus 954/2404 [40\%]; $\mathrm{p}<0.001$ ). Similarly, the proportion of "other" procedures performed decreased when comparing 2004 to 2005 and 2012 to $2013(598 / 2618$ [23\%] versus 681/3447 [20\%]; $\mathrm{p}=$ 0.004). Conversely, the proportion of adult reconstruction procedures increased between 2004 to 2005 and 2012 to 2013 (231/2618 [9\%] versus 648/3447 [19\%]; $\mathrm{p}<0.001)$. The proportion of trauma procedures remained similar when comparing the two times (599/2618 [23\%] versus $863 / 3447$ [25\%]; $\mathrm{p}=0.052$ ).

\section{Discussion}

As more residents pursue fellowship training, it is important to better investigate how this specialty training affects early-career practice patterns. Currently, there is limited evidence regarding the early-career practice patterns of fellowship-trained orthopaedic oncologists [15]. As the incidence of musculoskeletal tumors has remained relatively unchanged, appropriately allocating resources, including a strategy to optimize the number of tumor fellowship positions, to provide accessible, quality care is 
important at the national and societal levels. The current study used the ABOS Part II database and found that, although the number of ABOS Part II examinees with a history of orthopaedic oncology fellowship training is increasing, the median number of oncology-specific procedures performed by these fellows in independent practice has decreased. Furthermore, tumor procedures account for less than $50 \%$ of the overall procedure volume for the majority of tumor-trained fellows submitting cases for the ABOS Part II examination. Understanding these trends and general practice patterns is important to set realistic expectations for orthopaedic surgery residents considering a career in orthopaedic oncology. Although the results of our study cannot be generalized to the entire field of orthopaedic oncology, the trend of decreased subspecialty procedures with time, at least for surgeons during the first years of their career, is notable and should stimulate further discussion regarding the ideal approach to education and distribution of specialty care.

Our study does have several limitations. In addition to the limitations inherent to a database reliant on coding accuracy, we did not exclude examinees who reported more than one fellowship training experience. As such, despite orthopaedic oncology fellowship training, an individual's practice goals may not coincide with what we assumed to be a practice focused on orthopaedic oncology. However, we did take this into consideration and separately analyzed the subset of candidates who reported more than one fellowship training experience. In the ABOS Part II database, a primary CPT code is not specified for each procedure. Although we used ICD-9 codes to help clarify conflicting CPT codes for accurate procedural categorization, we erred on the side of listing a procedure as a tumor procedure when there was any question. This would only serve to inflate the proportion of tumor procedures performed by our cohort, which, in light of the presented findings, seems unlikely and, at worst, presents a generous estimate of tumor procedure volume. As previously mentioned, the results of our study reflect the practice patterns of orthopaedic surgeons with orthopaedic oncology fellowship training early during their career and may not be consistent with the scope of practice for more-experienced surgeons. We investigated ABOS Part II candidates with a history of orthopaedic oncology fellowship training. As we did not investigate other areas of subspecialty training, it unclear whether these trends would be seen in other subspecialty areas when using our methodology, although previous research would suggest a trend toward increasing subspecialization [9].

The first goal of our study was to investigate the number of examinees with a history of orthopaedic oncology fellowship training who submitted cases for the ABOS Part II examination. Although the time between completion of fellowship training and sitting for the ABOS Part II examination may vary, this served as a surrogate for the number of recently trained orthopaedic oncology fellows. Similar to previous studies [8,9], we found that there was a greater number of examinees who reported orthopaedic oncology fellowship training during the second half of the study than during the first 5 years of the study. Since the first orthopaedic oncology fellowship was established in the late 1970s, there has been a slow but steady increase in the number of available fellowship positions $[5,6]$. In a study also using the ABOS Part II database, Horst et al. [9] found that the overall number of Part II examinees reporting fellowship training increased from $76 \%$ in 2003 to $90 \%$ in 2013. Our study, and that of Horst et al., identified a consistent increase in subspecialty fellowship training in more recent years.

Interestingly, we found that nearly $24 \%$ of examinees with tumor fellowship training reported additional subspecialty training, similar to a previously published report [18], and this proportion was consistent during the entire study period. Horst et al. [9] investigated 7862 ABOS Part II candidates and excluded any candidate who reported multiple fellowships. Extrapolating on these numbers, we can estimate that $2.3 \%$ of all candidates, and $2.7 \%$ of candidates who pursued additional fellowship training, completed training in more than one subspecialty. This is a decidedly lower proportion than our results would indicate in orthopaedic oncology. Although the reasons are speculative, it could be attributable to factors such as candidates deciding that their clinical interest required training in more than one field, pursuit of an employment opportunity predicated on multiple fellowship training, or an anticipated volume of tumor procedures that would not provide sufficient procedure volume to fill a practice. A higher proportion of candidates with multiple fellowships may explain some of the lower proportion of tumor cases. However, based on our analysis, we do not believe that this finding alone accounts for the overall low oncologyspecific case volume in this cohort.

Another goal of our study was to describe the proportion of cases performed by recently trained orthopaedic oncologists using the ABOS Part II database. We found that for any given year, rarely did tumor procedures make up greater than $50 \%$ of the procedure volume for examinees with tumor fellowship training. In fact, in only 3 years, 2005,2009 , and 2012, did the majority of recently trained tumor fellows report that tumor procedures accounted for $50 \%$ or more of their overall practice volume. This is in contrast to a previous report suggesting that tumor procedures make up more than $70 \%$ of the clinical practice volume for members of the MSTS [18]. This difference may be explained by practice maturity, as ABOS Part II candidates are frequently early in their practice, which may 
not be indicative of what will transpire with more years in practice. In addition, there is a suspicion that candidates are "selective" in what procedures they actually perform during their board collection period so that the reported figures could underestimate what surgeons will actually do once the board collection period has been completed. Alternatively, White et al. [18] relied on surgeon estimation of the proportion of oncology-specific cases rather than case log data, and it is plausible that it is not an accurate account of their true practice. More recently, Miller et al. [15] found that tumor procedures accounted for $56 \%$ of the procedure volume for early-career orthopaedic oncologists while noting a high degree of variability. The cohort analyzed by Miller et al. likely differs from the current cohort, because participants were self-selected practitioners who reported 2 years of personal case lists. Our study focused on a database analysis of all surgeons with a history of orthopaedic oncology fellowship training, which is potentially more representative of recently tumor-trained orthopaedic surgeons as a whole. Although methods prevent direct comparison of the limited studies on this topic, a tumor surgeon's practice likely evolves with time, and practice patterns of early-career orthopaedic oncologists likely do not mirror those of their more senior colleagues. Our findings differ from those reported by Horst et al. [9], who reported that tumortrained Part II ABOS candidates from 2003-2013 performed more than $70 \%$ of procedures in their fellowship training. This discrepancy can be explained by methodologic differences, as Horst et al. considered any procedure that an applicant may have had exposure to during their fellowship training as specialty-specific. This included procedures such as total joint replacement, fracture fixation, and arthroscopy, which the candidate may have performed in the setting of a nonneoplastic condition. We believe that our methodology, specifically requiring an associated ICD-9 code with a tumor diagnosis for procedures that did not clearly involve a mass excision, presents a more accurate representation of procedures relevant to specialty training in orthopaedic oncology.

The final aim of our study was to describe changes in practice patterns during the 10-year period of the study. We found that the proportion of tumor procedures performed during the most recent years of the study significantly decreased compared with the earliest years of the study, whereas the proportion of adult reconstruction procedures significantly increased during the same time. Additionally, there was a general decrease in the median number of tumor procedures performed by each examinee during the most recent years of the study. Previous literature has suggested that the practices of orthopaedic surgeons have recently become more subspecialized [9]. Although this may be the case for the majority of fellowship-trained orthopaedic surgeons, the results of our study would suggest that this trend does not hold true when evaluating the proportion of tumor procedures performed by examinees with a history of orthopaedic oncology fellowship training. Additionally, the proportion of adult reconstruction procedures performed by these same examinees has more than doubled during the duration of the study, which may be at least partly attributable to the increased demand for lower extremity arthroplasty. We cannot tell if this is by choice of the surgeon or his or her practice partners or the result of the limited number of patients with tumor problems. It may be that oncology fellowship-trained surgeons prefer to take on challenging arthroplasty revision surgery to complement their tumor practice, which could affect the reported tumor cases. It has been well documented that the demand for THA and TKA has increased in recent years, with future projections anticipating a continued increase in volume $[12,13,16]$. The increased proportion of arthroplasty procedures by examinees with tumor fellowship training is consistent with this observation. This is important for trainees interested in orthopaedic oncology to understand, because joint replacement may represent a substantial proportion of their future clinical practice.

The last two findings of our study, that tumor procedures rarely make up more than $50 \%$ of total procedure volume and that the proportion of tumor cases performed by these same examinees has gradually decreased during the time of this study, is of particular interest given the recent focus on individual and hospital procedure volumes and clinical outcomes [2, 3]. Increased surgeon and hospital volume has been associated with decreased short-term morbidity and mortality after THA and TKA [4, 10, 11]. Although studying this relationship for orthopaedic oncology procedures is limited by relatively low annual procedure volumes, it is likely that procedural volumes influence perioperative complications for numerous unstudied surgical procedures. Because the overall volume of tumor procedures are much less than procedures like joint arthroplasty, even at high-volume cancer centers, it is possible that the discrepancies are not as dramatic as with more common procedures. Fellowship-trained oncologists who supplement their practice with arthroplasty cases and revisions might not suffer the "low-volume" phenomenon, and until studies are performed that show that local recurrence rates are increasing in association with lowvolume surgeons, we do not know if this is a factor for oncology. The theoretical risk of inferior outcomes, such as diminished survival, increased local recurrence, increased complications, or diminished function, for low-volume surgeons or hospitals must be balanced with the maintenance of adequate access to care for patients and the necessity of training and educating future surgeons. The question of the most appropriate number of tumor fellows 
to train and release in the work force is compelling and important. Our findings would suggest that the dilution of tumor-specific procedures may be explained primarily by the increase in the number of orthopaedic oncologists in recent years. However, this issue is unquestionably complex and likely dependent on numerous factors. A reduction in the number of tumor fellows trained may result in an increase in the proportion of tumor cases for each individual. Yet, it is not clear if this would have a positive effect, such as improved surgical outcomes, or negative effect, such as reduced access to regional care for patients in need of subspecialty treatment. A goal solely for increased access may have limitation as well, as the optimal treatment of sarcoma is best delivered at regional referral centers with a multidisciplinary team that specializes in the treatment of this rare cancer [14]. Perhaps the ideal scenario would be increasing the visibility and ease of referral to regional sarcoma centers rather than providing orthopaedic oncologists to communities without adequate multidisciplinary support. In addition, there are institutional factors to consider, as most tumor fellows are instrumental in facilitating and coordinating care for patients who often require a multidisciplinary approach. From a societal perspective, this issue is not easily resolved, as any movement toward restricting the number of fellowship positions offered may stimulate litigation on the grounds of restraint-of-trade. Given the findings of our study, it is clear that this is a complex topic that should be investigated more thoroughly, with input from societal leaders, graduate medical education representatives, and fellowship directors.

We found that the number of ABOS Part II examinees with a history of orthopaedic oncology fellowship training has increased during recent years. Although recent literature suggests that fellowship-trained orthopaedic surgeons are becoming more subspecialized with respect to the proportion of procedures performed in an individual subspecialty [9], we found that only one-third of examinees who report orthopaedic oncology fellowship training have practices with tumor procedures accounting for more than $50 \%$ of their total case volume. Additionally, the proportion of tumor procedures performed by the cohort as a whole and the median number of tumor procedures performed by an individual examinee have decreased during the 10-year period of this study. The findings of our study have several important and broad potential implications at the national and societal levels, and for the care of individual patients, that deserve further study. Specifically, the recent increase in the annual number of tumor-trained fellows coupled with the decreased proportion of tumor cases performed during the same time raises the question of "right-sizing" orthopaedic oncology fellowship training with the goal of optimizing accessibility to specialty care while maintaining the best possible surgical result. Although we do not place a value on these findings and do not know if this is a harmful or beneficial trend, this information should be of interest to current trainees interested in orthopaedic oncology fellowship training and to orthopaedic oncology educators. Based on these findings, future studies should investigate the evolution of practice patterns for fellowship-trained orthopaedic oncologists and the association between surgeon and hospital volume and oncology outcomes after musculoskeletal tumor procedures.

Acknowledgments We thank the ABOS and their staff for access to data and assistance during manuscript preparation. We also thank Yubo Gao PhD (Department of Orthopaedics and Rehabilitation, University of Iowa) for his statistical review.

\section{References}

1. Anglen JO, Weinstein JN; American Board of Orthopaedic Surgery Research Committee. Nail or plate fixation of intertrochanteric hip fractures: changing pattern of practice. A review of the American Board of Orthopaedic Surgery database. J Bone Joint Surg Am. 2008;90:700-707.

2. Birkmeyer JD, Siewers AE, Finlayson EV, Stukel TA, Lucas FL, Batista I, Welch HG, Wennberg DE. Hospital volume and surgical mortality in the United States. $N$ Engl $J$ Med. 2002;346:1128-1137.

3. Birkmeyer JD, Stukel TA, Siewers AE, Goodney PP, Wennberg DE, Lucas FL. Surgeon volume and operative mortality in the United States. N Engl J Med. 2003;349:2117-2127.

4. Bozic KJ, Maselli J, Pekow PS, Lindenauer PK, Vail TP, Auerbach AD. The influence of procedure volumes and standardization of care on quality and efficiency in total joint replacement surgery. J Bone Joint Surg Am. 2010;92:2643-2652.

5. DiCaprio MR. Oncology fellowships. Clin Orthop Relat Res. 2006:449:232-234.

6. Enneking WF. An abbreviated history of orthopaedic oncology in North America. Clin Orthop Relat Res. 2000;374:115-124.

7. Gaskill T, Cook C, Nunley J, Mather RC. The financial impact of orthopaedic fellowship training. $J$ Bone Joint Surg Am. 2009;91:1814-1821.

8. Hariri S, York SC, O’Connor MI, Parsley BS, McCarthy JC. Career plans of current orthopaedic residents with a focus on sexbased and generational differences. J Bone Joint Surg Am. 2011;93:e16.

9. Horst PK, Choo K, Bharucha N, Vail TP. Graduates of orthopaedic residency training are increasingly subspecialized: a review of the American Board of Orthopaedic Surgery Part II database. J Bone Joint Surg Am. 2015;97:869-875.

10. Katz JN, Barrett J, Mahomed NN, Baron JA, Wright RJ, Losina E. Association between hospital and surgeon procedure volume and the outcomes of total knee replacement. J Bone Joint Surg Am. 2004;86:1909-1916.

11. Katz JN, Losina E, Barrett J, Phillips CB, Mahomed NN, Lew RA, Guadagnoli E, Harris WH, Poss R, Baron JA. Association between hospital and surgeon procedure volume and outcomes of total hip replacement in the Unitd States Medicare population. $J$ Bone Joint Surg Am. 2001;83:1622-1629.

12. Kurtz S, Ong K, Lau E, Mowat F, Halpern M. Projections of primary and revision hip and knee arthroplasty in the United States from 2005 to 2030. J Bone Joint Surg Am. 2007;89:780785. 
13. Kurtz SM, Ong KL, Lau E, Bozic KJ. Impact of the economic downturn on total joint replacement demand in the United States: updated projections to 2021. J Bone Joint Surg Am. 2014;96:624-630.

14. Mankin HJ, Mankin CJ, Simon MA. The hazards of the biopsy, revisited. For the members of the Musculoskeletal Tumor Society. J Bone Joint Surg Am. 1996;78:656-663.

15. Miller BJ, Rajani R, Leddy L, Carmody Soni EE, White JR; Musculoskeletal Oncology Research Initiative. How much tumor surgery do early-career orthopaedic oncologists perform? Clin Orthop Relat Res. 2015;473:695-702.
16. Nho SJ, Kymes SM, Callaghan JJ, Felson DT. The burden of hip osteoarthritis in the United States: epidemiologic and economic considerations. J Am Acad Orthop Surg. 2013;21(suppl 1):S1-6.

17. Salsberg ES, Grover A, Simon MA, Frick SL, Kuremsky MA, Goodman DC. An AOA Critical Issue. Future physician workforce requirements: implications for orthopaedic surgery education. J Bone Joint Surg Am. 2008;90:1143-1159.

18. White J, Toy P, Gibbs P, Enneking W, Scarborough M. The current practice of orthopaedic oncology in North America. Clin Orthop Relat Res. 2010;468:2840-2853. 